DOI: 10.12731/2227-930X-2020-3-165-172

УДК 004.94

\title{
УПРАВЛЕНИЕ ПЕРЕВОЗКАМИ ЗАПАСНЫХ ЧАСТЕЙ ТРАНСПОРТНЫХ СРЕДСТВ
}

\author{
Преображенский Ю.П., Кострова В.Н., \\ Плотников А.А.
}

В статье рассматривается задача, связанная с поставками запасных частей транспортных средств. Решение ее базируется на имитационном подходе. Приведены результаты проведенного моделирования.

Ключевые слова: система перевозок; система поставок; управление; моделирование.

\section{CONTROL OF TRANSPORTATION OF SPARE PARTS OF VEHICLES}

\section{Preobrazhenskiy Yu.P., Kostrova V.N., Plotnikov A.A.}

The paper discusses the problem associated with the supply of spare parts of vehicles. Its solution is based on a simulation approach. The results of the simulation are presented.

Keywords: transportation system; supply system; management; modeling.

\section{Введение}

В настоящее время идет развитие организаций, связанных с производством и распространением запасных частей транспортных средств. При этом для решения задач на практике могут быть использованы разные методы, в том числе - имитационные. В данной работе рассматриваются возможности построения соответствующих моделей и получения на их основе результатов. 


\section{Особенности имитационной модели для эффективно-} го управления перевозками запасных частей транспортных средств. Разработка данной модели производится учетом различных специфических ситуаций, таких как: случайные временные распределения объемов перевозок для реализации работ по техническому обслуживанию и ремонту транспортных средств.

Запасные части в данной работе, если говорить об общих случаях мы будем определять на базе рекуррентных соотношений, как описано с следующем выражении (1):

$$
Z_{n+1}=Z_{n}+\eta_{n+1}-f\left(Z_{n+1}+\eta_{n+1}+\xi_{n+1}\right)
$$

В данном выражении введены такие обозначения:

$\xi_{n+1}-$ данное выражение определяет необходимое для перевозок количество запасных частей в соответствии со следующим временным интервалом - $(n, n+1] ; \eta_{n+1}$ - данное выражение определяет количество запасных деталей, которые были перевезены в соответствии со следующим временным интервалом - n+1; $f\left(Z_{n+1}+\eta_{n+1}, \xi_{n+1}\right)$ данное выражение определяет функционал оценки задействованного количества запасных частей на $\mathrm{n}+1$ данный момент времени.

Если рассматривать наиболее простую имитационную модель, то предполагается, что количество запасных частей, которые были перевезены, представляет из себя совокупность независимых случайных величин, которые при этом имеют одинаковое распределение. Что касается перевозок, то здесь подразумевается использование заранее разработанной политики по управлению поставками и для функции f, которая в свою очередь формирует ту самую политику управления при помощи следующего выражения (2):

$$
f\left(Z_{n+1}+\eta_{n+1}, \xi_{n+1}\right) \leq \xi_{n+1}
$$

На этапе 1-го разделения моделей по управлению запасами на складе запасных частей транспортных средств возможно выполнение и не выполнение неравенства

$$
f\left(Z_{n+1}+\eta_{n+1}, \xi_{n+1}\right)>Z_{n}+\eta_{n+1}
$$

В случае если наблюдается задолженность, то в этом случае, мы имеем следующую функцию: $f\left(Z_{n+1}+\eta_{n+1}, \xi_{n+1}\right)=\xi_{n+1}$, и неравенство, рассмотренное выше (3), принимает иной вид (4): 


$$
Z_{n+1}=Z_{n}+\eta_{n+1}-\xi_{n+1}
$$

здесь отрицательное значение количества запасных деталей на складе означает что, в данное время мы можем наблюдать задолженность, которая соответствует противоположному значению. Для определения задолженности с учетом $\mathrm{n}+1$ используется следующее выражение (5):

$$
B_{n+1}=\max \left(0,-Z_{n+1}\right)=-\min \left(0, Z_{n}+\eta_{n+1}-\xi_{n+1}\right)
$$

В случаи не допустимости задолженности, поступившие заявки могут быть выполнены, только в случае если на складе имеется детали, которые были запрошены для перевозки, и тогда мы используем следующее равенство (6):

$$
f\left(Z_{n+1}+\eta_{n+1}, \xi_{n+1}\right)=-\min \left(Z_{n}+\eta_{n+1}, \xi_{n+1}\right)
$$

Тогда, приведенное выше неравенство (3), преобразуется в следующий вид:

$$
Z_{n+1}=-\max \left(0, Z_{n}+\eta_{n+1}, \xi_{n+1}\right)
$$

Приведенная выше стратегия управления запасами на складе при помощи соответствующих перевозок, дает возможность к появлению дефицита, соотношение которого можно задать (на $\mathrm{n}+1$ данный момент времени), при помощи следующего выражения (8):

$$
D_{n+1}=\xi_{n+1}-f\left(Z_{n+1}+\eta_{n+1}, \xi_{n+1}\right)=-\min \left(0, Z_{n}+\eta_{n+1}-\xi_{n+1}\right)
$$

Так же предлагается ввести в процесс моделирования процессами перевозок стратегию по управлению запасами на складе в виде: $\left(Z_{\text {min }}\right.$, $Z_{\text {max }}$ ), данный тип стратегии, как видно из приведенного обозначения всего два действительных числа: $Z_{\text {min }}$ и $Z_{\text {max }}$ имеющие следующее неравенство: $0 \leq Z_{\min } \leq Z_{\max }<\infty$. Данная стратегия предполагает, что заявка на перевозки запасных частей для транспортных средств должна быть выполнена полностью, т.е. в момент превышения уровня запаса над уровнем объема заказа указанного в заявке для осуществления перевозок. Однако, если количество запасных частей транспортных средств на складе становится меньше заданной отметки $Z_{\text {min }}$, то на данный промежуток времени заказ тут же проходит стадию реализации, объем которого приводит уровень запаса до значения $Z_{\max }$.

Таким образом, провести расчет объема заказа можно на основе следующего выражения (9): 


$$
\eta_{n+1}=\left\{\begin{array}{c}
0, Z_{\min } \leq Z_{n} \leq Z_{\text {max }} \\
S-Z_{n}, \quad Z_{n}<Z_{\text {min }}
\end{array} .\right.
$$

В итоге, приведенное раннее неравенство (3), преобразовывается в следующее равенство (10), это равенство справедливо лишь для данной стратегии:

$$
Z_{n+1}=\left\{\begin{array}{l}
Z_{n}-\xi_{n+1}, \quad Z_{\min } \leq Z_{n} \leq Z_{\max } \\
Z_{\max }-Z_{n}-\xi_{n+1}, \quad Z_{n}<Z_{\min }
\end{array}\right.
$$

Следует отметить, что при использовании данной стратегии, заказанный объем запасных деталей для отопительного оборудования, проходит стадию реализации с небольшой задержкой.

Для формирования монотонной стратегии по управлению перевозками к складу, следует использовать задания попарного значения $x^{*}$.

Но, при этом осуществление заказа на перевозку не производится при наличии данного количества запасных частей. В случаи $Z_{n}<x^{*}$ то заявка на перевозку производится в тот же момент, однако, пополнение происходит на случайный объем запасных частей $X_{n+1}$.

Таким образом, мы получаем следующую рекуррентную схему:

$$
Z_{n+1}=\left\{\begin{array}{c}
Z_{n}+X_{n+1}-\xi_{n+1}, Z_{n}<x^{*} \\
Z_{n}-\xi_{n+1}, Z_{n} \geq x^{*}
\end{array},\right.
$$

При данной схеме пополняемое при перевозках количество запасных деталей, как и заявка на них $\xi_{n+1}$, являются случайными величинами.

Разработанная имитационная модель для управления процессами перевозок запасных частей транспортных средств дает нам возможность различным образом манипулировать приведенными стратегиями по управлению запасами на складе. То есть мы можем проводить манипуляции с объемами перевозок, где времена поставок в общем случае являются случайными величинами, в отношении которых проводится подборка распределений с указанной заранее дисперсией и характеристиками математического ожидания.

Параметры имитационной модели. Представленные ранее рекуррентные схемы являются базовыми, и имеют однородную па- 
раметрическую форму, таким образом они создают некую однопараметрическую модель, целью которой является эффективное управление перевозками к складу запчастей транспортных средств.

Рассмотрим параметры, которые были включены в разработанную модель:

1. минимальное количество запасов запчастей транспортных средств на складе для формирования заявки на их поставку $\left(Z_{\text {min }}\right)$;

2. максимальное количество запасов на складе запчастей транспортных средств для формирования заявки на их поставку $\left(Z_{\text {max }}\right)$;

3. переменная, при помощи которой задается ранее не определенное время для реализации поставки запчастей транспортных средств, т.е. случайное время, при котором изначальное распределение имеет равные промежутки времени $\left(T_{z a k}\right)$;

4. полигон планирования системы управления запчастей транспортных средств на складе отопительного оборудования (T);

5. определение интенсивности запасов на отдельную запасную часть транспортных средств $(\mu)$;

6. стоимость хранения одной запчасти транспортных средств на складе, относительно единицы времени $(s)$;

7. стоимость за поставку одной партии $(g)$;

8. размер штрафа за нехватку запасных частей и др. $(h)$.

Результаты проведенного моделирования. При анализе работоспособности предложенного алгоритма минимальное и максимальное количество запасов запчастей отличалось в 5 раз. Количество итераций при работе алгоритма не превышало 18 . Не определенное время для реализации поставки запчастей транспортных средств было равно 1 часу. В результате проведенного имитационного моделирования в результате перевозок неравномерность распределения запчастей по складам не превышало $10 \%$.

\section{Вывод}

Таким образом, в результате проведенного анализа показана возможность построения алгоритма для имитационного модели- 
рования перевок запчастей транспортных средств. Приведены некоторые результаты работы алгоритма.

Информация о конфликте интересов. Авторы заявляет об отсутствии конфликта интересов.

Информация о спонсорстве. Исследование не имело спонсорской поддержки.

\section{Список литературь}

1. Берман Н.Д., Белов А.М. Общественный транспорт и инновации // International Journal of Advanced Studies. 2019. T. 9. № 2. C. 7-13.

2. Шакиров А.А., Зарипова Р.С. Особенности моделирования логистических систем // International Journal of Advanced Studies. 2019. T. 9. № 4. C. 27-31.

3. Сапожникова С.М. Корпоративное управление в железнодорожном транспорте // International Journal of Advanced Studies. 2019. T. 9. № 4. C. 19-42.

4. Преображенский Ю.П. Информационные технологии, используемые в сфере менеджмента // Вестник Воронежского института высоких технологий. 2018. № 2 (25). С. 43-46.

5. Преображенский А.П. Возможности обеспечения развития предприятий // В мире научных открытий. 2015. № 10 (70). С. 196-201.

6. Гостева Н.Н., Гусев А.В. О возможности увеличения эффективности производства // Вестник Воронежского института высоких технологий. 2017. № 1 (20). С. 76-78.

7. Львович И.Я., Преображенский А.П., Чопоров О.Н. Использование информационных систем в управлении производством // Научный взгляд в будущее. 2018. Т. 3. № 9. С. 94-98.

8. Преображенский А.П., Чопоров О.Н. Построение многокритериальной модели работы предприятия // Наука Красноярья. 2017. Т. 6. № 3-4. С. 183-188.

\section{References}

1. Berman N.D., Belov A.M. Obshchestvennyj transport i innovacii // International Journal of Advanced Studies. 2019. T. 9. № 2. S. 7-13. 
2. Shakirov A.A., Zaripova R.S. Osobennosti modelirovaniya logisticheskih sistem // International Journal of Advanced Studies. 2019. T. 9. № 4. S. 27-31.

3. Sapozhnikova S.M. Korporativnoe upravlenie v zheleznodorozhnom transporte // International Journal of Advanced Studies. 2019. T. 9. № 4. S. 19-42.

4. Preobrazhenskij Yu.P. Informacionnye tekhnologii, ispol'zuemye v sfere menedzhmenta // Vestnik Voronezhskogo instituta vysokih tekhnologij. 2018. № 2 (25). S. 43-46.

5. Preobrazhenskij A.P. Vozmozhnosti obespecheniya razvitiya predpriyatij // V mire nauchnyh otkrytij. 2015. № 10 (70). S. 196-201.

6. Gosteva N.N., Gusev A.V. O vozmozhnosti uvelicheniya effektivnosti proizvodstva // Vestnik Voronezhskogo instituta vysokih tekhnologij. 2017. № 1 (20). S. 76-78.

7. L'vovich I.Ya., Preobrazhenskij A.P., Choporov O.N. Ispol'zovanie informacionnyh sistem $\mathrm{v}$ upravlenii proizvodstvom // Nauchnyj vzglyad v budushchee. 2018. T. 3. № 9. S. 94-98.

8. Preobrazhenskij A.P., Choporov O.N. Postroenie mnogokriterial'noj modeli raboty predpriyatiya // Nauka Krasnoyar'ya. 2017. T. 6. № 3-4. S. 183-188.

\section{ДАННЫЕ ОБ АВТОРАХ}

Преображенский Юрий Петрович, доцент, кандидат технических наук, доцент

Автономная некоммерческая образовательная организачия выстего образования Воронежский институт высоких технологий

ул. Ленина, 73а, г. Воронеж, 394043, Россия

gorbenkoon@yandex.ru

Кострова Вера Николаевна, профессор, доктор технических наук, профессор

Федеральное государственное образовательное учреждение выстего образования Воронежский государственный технический университет 
ул. 20 лет Октября, 84, г. Воронеж, 394006, Россия gorbenkoon@yandex.ru

Плотников Александр Алексеевич, доцент, кандидат технических наук, доцент

Автономная некоммерческая образовательная организачия выстего образования Воронежский институт высоких технологий

ул. Ленина, 73а, г. Воронеж, 394043, Россия

gorbenkoon@yandex.ru

\section{DATA ABOUT THE AUTHORS}

Preobrazhenskiy Yuriy Petrovich, associate professor, doctor of technical sciencies, associate professor Voronezh Institute of high technologies 73a, Lenin Str., Voronezh, 394043, Russia gorbenkoon@yandex.ru

Kostrova Vera Nikolaevna, Professor, doctor of technical Sciences, Professor Federal state educational institution of higher professional education Voronezh state technical University 84, 20 years of October Str., Voronezh, 394006, Russia gorbenkoon@yandex.ru

Plotnikov Alexander Alexeevich, associate Professor, Candidate of Technical Sciences, Associate Professor Voronezh Institute of high technologies 73a, Lenin Str., Voronezh, 394043, Russia gorbenkoon@yandex.ru 